CORRECTION

https://doi.org/10.1038/s41586-018-0247-3

\title{
Publisher Correction: Integrating photonics with silicon nanoelectronics for the next generation of systems on a chip
}

Amir H. Atabaki, Sajjad Moazeni, Fabio Pavanello, Hayk Gevorgyan, Jelena Notaros, Luca Alloatti, Mark T. Wade, Chen Sun, Seth A. Kruger, Huaiyu Meng, Kenaish Al Qubaisi, Imbert Wang, Bohan Zhang, Anatol Khilo, Christopher V. Baiocco, Miloš A. Popović, Vladimir M. Stojanović \& Rajeev J. Ram

Correction to: Nature https://doi.org/10.1038/s41586-018-0028-z, published online 18 April 2018.

In this Letter, owing to an error during the production process, the author affiliations were listed incorrectly. Affiliation number 5 (Colleges of Nanoscale Science and Engineering, State University of New York (SUNY)) was repeated, and affiliation numbers 6-8 were incorrect. In addition, the phrase "two oxide thickness variants" should have been "two gate oxide thickness variants". These errors have all been corrected online. 